\section{§9. Stable Sheath Formation in Expanding Magnetic Field to Divertor Plate}

Tomita, Y., Takayama, A., Takamaru, H., Sato, T.

The stable sheath formation in expanding magnetic field to a divertor plate was studied theoretically by onedimensional analysis. In fusion devices the magnetic field is expanding in the direction of the divertor plate, i.e. the magnitude of magnetic field is decreasing to the plate. In this configuration ions are accelerated to the plate due to the gradient of the magnetic field strength, so called a mirror force. The bombardment of accelerated ions to the plate may cause several severe problems to fusion plasmas, for example, release of large amount of impurities from the divertor plate. Limited research efforts have been carried out describing magnetic field effects on various potential formation and particle and heat fluxes to the divertor plate. The plasma-wall interaction in an oblique to the plate but uniform magnetic field has been studied by means of 1D-PIC numerical simulation.1) This analysis shows the formation of a quasi-neutral magnetic pre-sheath preceding the electrostatic Debye sheath, which scales to the ion gyroradius at the sound speed and to the incidence angle of the magnetic field. Sato 2) clarifies this magnetic pre-sheath is attributed to the ion polarization drift by the two dimensional kinetic analysis. None of effects, however, of non-uniformity of the magnetic field has been taken into account on the stable electrostatic potential and sheath formation. In this report, we consider a collisionless sheath model between an infinite metal plate and a quasi-neutral plasma in the expanding magnetic field to the plate.

The one dimensional analysis gives the the condition for the stable Debye sheath formation in expanding magnetic field to the mean ion velocity at the plasmasheath boundary $\left\langle v_{z}\right\rangle_{b}$ :

$$
<v_{z}^{-2}>_{b}^{-1} \geq \frac{Z_{T_{e}} / M}{1-\alpha},
$$

the parameter $\alpha$, which indicates the effect of a nonuniform magnetic field, is defined:

$$
\left.\alpha \equiv \frac{T_{e}}{e}\left(1+\frac{1}{2}<v_{\perp}^{2} / v_{z}^{2}>_{b}\right) \frac{d(\ln B) / d z}{d \phi / d z}\right|_{b} .
$$

The subscript $b$ indicates the value at the plasma-sheath boundary. In our case, where the strength of magnetic field is decreasing to the wall as well as electrostatic potential $\phi$, the parameter $\alpha$ has a value of positive definite, which means the generalized Bohm criterion for the case of nonuniform magnetic field is restricted compared to that of the uniform magnetic field. 3,4 )

$$
\left\langle v_{\approx}^{-2}>_{b}^{-1} \geq \frac{Z T_{e} / M}{1-\alpha} \geq \frac{Z_{T_{e}}}{M} .\right.
$$

The difference, however, between both cases is an order of the Debye length to a plasma radius, which is negligible small.

The requirement for the ion flow velocity inside the quasi-neutral plasma in order to form the stable pre-sheath is obtained from the condition of the quasi-neutrality.

$$
\begin{aligned}
& \frac{Z T_{e}}{M}<v_{\Sigma}^{-2}>\text { in }=\frac{1}{1-\delta_{s}}\left[1+\delta_{s} \frac{Z T_{e}}{T_{s}}\right. \\
& \left.-\left.\left(1-\delta_{s}\right) \frac{T_{e}}{e}\left(1+\frac{1}{2}<v_{\perp}^{2} / v_{s}^{2}>\text { in }\right) \frac{d(\ln B) / d z}{d \phi / d z}\right|_{i n}\right]
\end{aligned}
$$

where $\delta_{s}$ is defined by the ratio of the density of the source particle to the electron density : $\delta_{s} \equiv Z n_{i s} / n_{e}$. The values denoted by the subscript in is evaluated at the plasma injection point to the quasi-neutral plasma. The temperatures of electrons $T_{e}$ and source particles $T_{s}$ are assumed uniform inside the plasma region. The third term of RHS of eq.(4), which designates the non-uniformity of the magnetic field, is the order of unity because of the same scale length of the magnetic field and the electrostatic potential. On the other hand, the second term of RHS of eq.(4), which indicates the effects of the particle source, is much larger than unity because of higher plasma temperature compared to that of source particle. Therefore the averaged value of $\left\langle v \bar{z}^{-2}\right\rangle_{\text {in }}^{-1}$ at the injection point becomes much less than the ion sound speed $\left(Z T_{e} / M\right)$. In case of no plasma source $\left(\delta_{s}=0\right)$, the RHS of eq.(4) becomes less than unity, which implies the value of $\left\langle v \bar{z}^{-2}\right\rangle_{\text {in }}^{-1}$ is required larger than the ion sound speed at the injection boundary.

Including other phenomena, for example Coulomb collision and charge exchange process, is the future issue.

\section{References}

1) R. Chodura, J. Nucl. Mater. 111 \& 112 (1982) 420.

2) K. Sato, H. Katayama, and F. Miyawaki, Contrib. Plasma Phys. 34 (1994) 133.

3) D. Bohm, in The Characteristics of Electrical Discharges in Magnetic Fields, edited by A. Guthrie and R.K. Wakerling (McGraw-Hill, New York, 1949), Chap.4, p.77.

4) E.R. Harrison and W.B. Thompson, Proc. Phys. Soc. London 74 (1959) 145. 